\title{
THERAPIST: Towards an Autonomous Socially Interactive Robot for Motor and Neurorehabilitation Therapies for Children
}

\author{
L.V. Calderita and P. Bustos \\ C. Suárez Mejías \\ F. Fernández \\ A. Bandera \\ RoboLab \\ University of Extremadura \\ Cáceres, Spain \\ Grupo de Innovación Tecnológica, UCA
Hospital Universitario Virgen del Rocío \\ Sevilla, Spain \\ \{lvcalderita, pbustos\}@unex.es cristina.suarez.exts@juntadeandalucia.es \\ and Learning Group \\ University Carlos III \\ Madrid, Spain \\ ffernandez@uc3m.es \\ Grupo ISIS \\ University of Málaga \\ Málaga, Spain \\ ajbandera@uma.es
}

\begin{abstract}
Exploiting the use-dependent plasticity of our neuromuscular system, neuro-rehabilitation therapies are devised to help patients that suffer from injuries or diseases in this system, such as those caused by brain damage before or during birth or in the first years of life (e.g. due to cerebral palsy or obstetric brachial plexus palsy). These therapies take advantage of the fact that the motor activity alters the properties of our neurons and muscles, including the pattern of their connectivity, and thus their functionality. Hence, a sensor-motor treatment where the patient makes certain movements, will help her to (re)learn how to move the affected body parts. But this traditional rehabilitation processes come at a cost: therapies are usually repetitive and lengthy, reducing motivation and adherence to the treatment and thus limiting the benefits for the patients. This paper describes the motivation, experiences and current efforts towards the final development of THERAPIST, a socially interactive robot for neuro-rehabilitation assistance. Our starting hypothesis was that patients could get consistently engaged in a therapeutic non-physical interaction with a robot, facilitating the design of new therapies that should improve the patient recovery time and reduce the overall socio-economic costs. This hypothesis was validated by our initial experimental studies, which showed that pediatric patients can be easily driven into highly attentive and collaborating attitudes by letting them interact with a robot. However, in order to be safe and robust, this robot was teleoperated, requiring a great effort on supervision from clinic professionals. The development of a real socially interactive robot will require the intersection of multiple challenging directions of research that we are currently exploring.
\end{abstract}

\section{INTRODUCTION}

Neuro-rehabilitation therapy pursuits the recovery of damaged neuronal areas and/or muscles from the repetitive practice of certain motor or cognitive activities. The patient's recovery directly depends on the adherence to neuro-rehabilitation therapy. Conventional methods consisting of repetitions usually make the patient feel unmotivated and neglect complying with the appropriate treatments. In addition, the treatment of these motor and cognitive deficits requires intensive and extended rehabilitation sessions that demand sustained dedication and effort by professionals and incur in accretive costs for the institutions. Robotic Science has become in recent years a useful tool to address these issues. For instance, the SCRIPT (http://scriptproyect.eu) project, funded by the EU 7th FP, includes a work package for motivational rehabilitation. The aim is to develop novel techniques to guide and encourage patients to ensure the process is as intuitive and interesting as possible. As other initiatives, this project focuses on telerobotic procedures, a strategy that ensures safety and robustness in a critical scenario. With the aim of increasing the robot autonomy, one of the most active research fields in this topic is the design of socially assistive robots [11]. These robots can be used in non-contact, hands-off therapeutic interactions with the patient, exploiting embodiment, emotions, dialogues, personality, user models and socially situated-learning. They may provide cost-effective solutions to the need of extended and dedicated one-on-one care, and also to monitor progress during physical therapy and daily life, providing tireless motivation, encouragement and guidance.

From pioneering systems such as JavaTherapy [12], the application of computer-assisted technologies to rehabilitation has generated a positive feedback from therapists and an increasing demand of solutions that will put the emphasis on motivation through entertainment. For instance, the ArmeoSpring Pediatric from Hocoma (http://www.hocoma.com/) is a robotic tool to improve therapy by facilitating intensive and functional movement exercises. This tool supports the therapy by motivating, game-like tasks. However, given the inherent people tendency to engage with life-like social behavior, the use of the robot for augmenting or maintaining the patient's motivation provides an important advantage over game-based approaches [5]. This paper describes our own experience on the definition of a new neuro-rehabilitation therapy where a robot is used for hands-off interaction. In this kind of applications, the role of physical embodiment, the capacity of responding to new events and the ability for unfolding multiple handsoff interaction strategies (e.g. speech or facial expressions) are fundamental topics. In order to fulfill these requirements, we argue that the robot should be endowed with a robust and efficient cognitive architecture. Here, we describe the internal software architecture for THERAPIST, a robot that will perform as an innovative trainer in motor deficit therapies. THERAPIST will be a socially interactive robot, and therefore it must be endowed with cognitive functionalities that will allow it to operate as an autonomous, active assistant. To achieve these social behaviors, which will provide an efficient and friendly human-robot interaction, the cognitive architecture proposed on this paper is based on the combination of an inner-simulation engine, automated planning, machine learning 


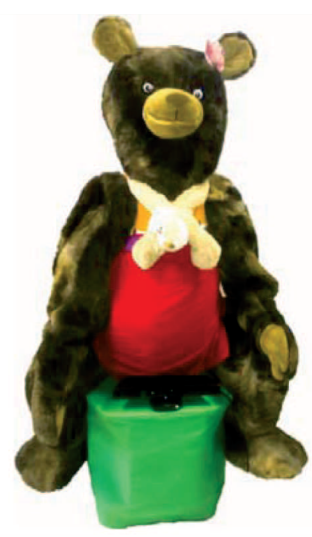

Fig. 1. The robot URSUS

and specialized skills. This architecture will contribute to the integration of the perceived information with decision-making processes, monitoring of activities and high-level task replanning, with the main goal of improving the social skills of THERAPIST and its ability to help in rehabilitation therapies. The rest of the paper is organized as follows: Section 2 describes the motivation and main guidelines of the internal cognitive architecture for THERAPIST. Section 3 draws the conclusions and future work.

\section{From URsus to Therapist: TOWARdS A Socially ASSISTIVE ROBOT}

In 2009, the project ACROSS was launched. It is a Singular Strategic Scientific-Technological project financed by the Spanish government under the Plan Avanza initiative whose main aim is to incorporate robotic services into social scenarios. In this project, the research groups of the University of Extremadura (UEx, RoboLab) and of the Hospital Universitario Virgen del Rocío (HUVR) worked together on the development of a robot that helps patients and therapists in the realization of repetitive rehabilitation exercises. The robot was named URSUS (see Fig.1) and currently is a semiautonomous robot, equipped with a RGBD sensor to monitor the patient's movements. More information on its hardware and therapeutic abilities can be found in [13]. Although URSUS has proven that the patient's predisposition to their neurorehabilitation treatments can be improved, its internal control architecture needs to be redefined at all abstraction levels. Thus, at higher level, it encodes each therapeutic session as a hard state machine. Hence, there is not way for (re-)planning or learning new possibilities of actuation. This issue obliges the robot to work under human supervision, as new situations continuously appears. Furthermore, there is not an easy way to learn from these situations. On the other hand, at mid level, the social skills of URSUS are not yet fully developed. This can provoke that it arises some degree of deception inherent to the personification of the robot by the patients or caregivers [6]. Finally, it is needed to take into account several considerations about the physical appearance of URSUS. For instance, recent studies already demonstrate that the size of the robot has impact on the interaction and perceived role [6]. Although physical issues will be also reconsidered in the design of THERAPIST, we are currently involved in the development and validation of a novel cognitive architecture. This architecture

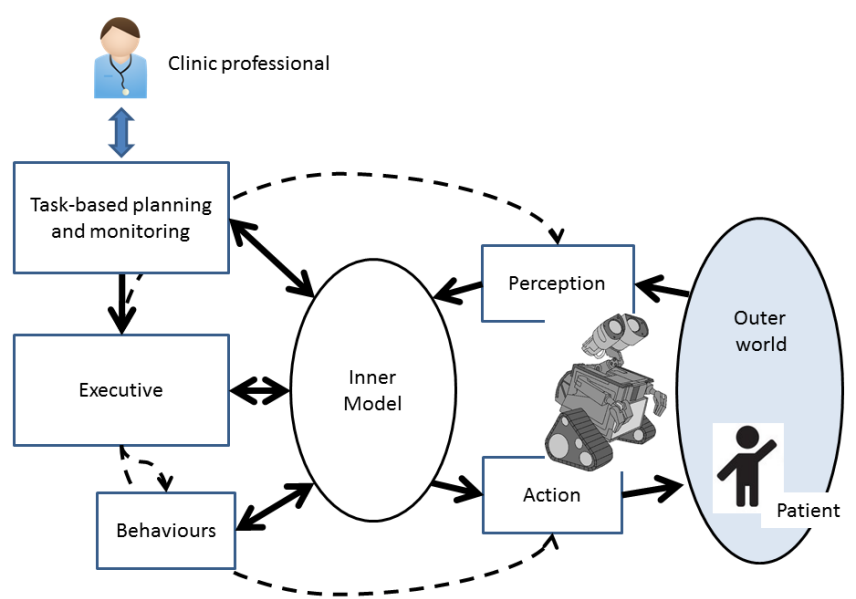

Fig. 2. An overview of the novel cognitive architecture for THERAPIST

will be the internal core of the robot. Figure 2 shows a schematic overview of the proposed cognitive architecture. Our final goal is that THERAPIST will be able to establish a relationship with the patient that leads toward intended therapeutic objectives. That is, the development of an effective socially assistive robot [6].

There are four main modules on Fig. 2. The 'Taskbased planning and monitoring' module is in charge of the application of Automated Planning technologies, together with Machine Learning, to the development of high level control components of THERAPIST. These components will allow an efficient interaction between the robot and patients. The Planning and Learning Architecture (PELEA) [1] will be our starting point, adding human-robot interaction facilities and requirements. The planning architecture will be communicated with the other modules through the Tasks, i.e. the units of the Plans, provided to the 'Executive' module, and through the Symbolic variables, updated at the 'Inner Model' by the 'Behaviours' (in the figure, these behaviors are classified as 'Actions', 'Perceptions' and 'Behaviours', but all of them can be grouped inside this last module). To make this connection, we should provide a complete description of how to map the high level components described in the planning language with the low level skills. In addition, mechanisms to permit the mapping among the high and low level elements of the architecture must also be included. The user (therapist) should be able to include new high level components (concepts, actions, etc) in the domain description, as well as to acquire new skills and connect the high level components with the skills. That will favor the inclusion of new therapies without the interaction of the developers, following a Programming by Demonstration approach. On the other hand, the 'Inner Model' module addresses the design and development of an internal representation (inner model) of the real world. This representation could be seen as a 3D geometrical view of the perceived space, including static environment elements and dynamic items such as the patient or the robot itself. The 'Executive' module is the responsible of launching each unit of the Plan. For this end, it will activate the necessary 'Behaviours'. These could work in a 'reactive' way, by subscribing 
to the outcomes of certain sensors or to the state representation at the 'Inner Model'. Thus, they will provide geometric data to update the 'Inner Model' (e.g. a Human Motion Capture or a Face Tracker) or generate symbolic data such as 'the patient is looking THERAPIST' or 'the patient is getting up of the chair'. These symbolic data will be managed using a specific component inside the 'Inner Model' module (e.g. the ORO server [9]). Other activated behaviors work in an active way, including an internal state machine or an specific planner (e.g. a conversational module). In the current version of the architecture, the actuation of the 'Executive' module is a priori established. That is, it will not be modified by the situation (e.g., by the emotional state of the patient or the degree of success on the execution of the therapy). The ability of modifying the current Plan is in charge of the 'Taskbased planning and monitoring' module. Next, we detail the motivation and design of the specific modules ('Task-based planning and monitoring', 'Executive' and 'Inner Model') of the architecture.

\section{A. Inner Model for Prediction, Evaluation and Selection}

In order to engage patients in social interactions, THERAPIST should be able to emanate responses at human interaction rates, and exhibit a pro-active behavior [7]. This pro-active behavior implies that the internal architecture of THERAPIST should not only be able to perceive and act. It should also be able to perform off-line reasoning. Cognition is the ability that allows us to internally deal with the information about the outer world and, hence, this ability is subject to the existence of an internal representation of this information. Although internal models and representations of the state of the external world were traditionally rejected by the reactive paradigms [3], recent works suggest that cognitive architectures cannot work on a passive, bottom-up fashion, simply waiting to be activated by external stimuli. Instead, these architectures must continuously use memory to interpret sensory information and predict the immediate future. These predictions about the outer world can be used to actively drive the resources to relevant data in top-down modes of behavior, allowing an efficient and accurate interpretation of the environment [4], [8]. Without this ability, the responses of the robot in this scenario will not be generated at the needed rate, driving patients and caregivers to deception [6]. As Fig. 2 shows, in our proposal, the deliberative planner will be built over a inner model, a set of parameterized structures and procedures that constitute a virtual representation of the reality. In the restricted scenario where our therapies are conducted, this model include a robot model, a patient model and a room model. This model should not be available only through interaction with the outer world, but it should be used as virtual reality by certain modules. The behaviors that built and update this Inner Model are fully developed and validated [2], [10]. Thus, we currently have an example of the situation postulated by Holland [8]: at the heart of the mechanism is not just the body in the environment, it is a model of the body in a model of the environment. This situation allows emanating predictions from the model, which can be correlated with real perceived information to drive attention, increase efficiency and filter noisy perception, while the contents of the mental model are also updated through experience.

\section{B. Deliberative level: Task-based Planning and Monitoring}

The Inner Model can perform short-scale predictions and evaluate the consequences of actions. These processes become harder to execute as the time horizon is more distant in the future. One possible solution is to translate the problem to a pure declarative domain, where a symbolic automated planner can reason about the truth conditions of predicates, in a timeless, crisp representation. These symbolic systems are especially well-suited for natural language understanding and interaction. The abstraction from geometric to symbolic terms is not straightforward, but can be simplified if the scenario is known in advance. Thus, our models of robot or human link geometric parts with symbolic names (left arm, head...). The room and the objects in it are also a priori known, so they can be also annotated (windows, doors...). In this situation, we can consider that the robot has an initial Model of the world, whose similarity with the real one is very high. Graph grammars can be used to avoid situations that cannot occur in this scenario, maintaining the consistency of the Inner Model. This strategy has been recently validated with success [10]. As aforementioned, conceptual information about the scenario will be managed using the ORO server [9]. We are also developing the ontology that stores this information. This ontology is defined according to the rules and constraints described in the Web Ontology Language (OWL) and the Resource Description Framework (RDF) specifications.

Then, the Inner Model is now able to provide a symbolic representation of the state whose degree of completeness is total, as models and grammar graphs are for instance able to provide coherent information about occluded parts of the body of the patient or about the position of parts or objects on the environment that are out of the current field of view of the robot. Over this representation, the Planning and Learning Architecture PELEA works. PELEA combines two planners, one for high level actions and one for low level ones, with modules in charge of plan monitoring, goals generation and selection, re-planning triggering, etc. It also includes machine learning modules. In THERAPIST, the PELEA architecture will occupy the initial block covering the planning and learning functionalities. We are extending its capabilities to include human-robot interaction (HRI) planning and human assisted learning of new therapies. Planning for HRI interactions is a new and exciting area of research that has only recently began to be explored, and that fills an urgent need specially in situations like the use cases we are evaluating. Furthermore, we are exploring the challenging situation in which the therapist creates new therapies and teaches them to the robot without the need of any additional programming.

\section{The Executive module}

Above the 'Behaviours' modules we can find the Executive. This element is placed at the interface between the Task-based Planning module and the behaviors and is tightly connected to the 'Inner Model' module. The goal of the Executive is to convert high-level symbolic plan steps into a set of concurrent low-level active behaviors, and to monitor their activity by subscribing to the perceptive events generated by them. As briefly described at Section 2, to achieve this, the Executive uses a structure that maps each plan step to a set of behaviors. 
For each plan action the Executive performs the following steps:

1) Check if the preconditions of the actions hold in the world representation

2) Select the behaviors that will materialize the required symbolic action

3) Activate them and subscribe to the perceptual events that they might generate

4) Wait until the completion of the task or until some event forces to interrupt the step. Any incoming events are whether ignored, transmitted to the 'Inner Model' representation or taken as a cause of interruption.

5) After an interruption, the Executive calls for replanning.

\section{DISCUSSION AND FUTURE WORK}

Rehabilitation robotics constitutes an emerging area of research, where the aim is to include robotics technology in the time-consuming and labor-intensive process associated to neuro-rehabilitation therapies. As in other fields of application, robots can offer several key advantages, such as the possibility to perform a consistent and personalized treatment without tiring, and its capacity to use sensors to acquire data, which can provide an objective quantification of the recovery. Moreover, apart from giving mechanical/physical assistance in rehabilitation, recent studies postulate that robotic technology can motivate and coach patients in the realization of the repetitive efforts that constitute the primary stimulus for recovery. Due to these reasons, one of the more active research challenges in rehabilitation robotics is the design and development of safe and effective human-robot interaction for hands-off, socially assistive robotics. Clinical experiments demonstrate that motivation is an important factor for successfully addressing a lengthy neuro-rehabilitation therapy and it is usually employed as a determinant of rehabilitation outcome. Hence, active engagement towards a therapy is typically equated with motivation. Within this context, socially assistive robots emerge as a new field of robotics whose aim is to develop systems that assist patients through social rather than physical interaction. They provide therapy oversight, coaching and motivation using the robot's abilities to interact and maintain the interest of patients. Furthermore, depending of the degree of autonomy of the robot, coaching and motivation can be provided with little supervision by professional therapists. It should be noted that it is typically pointless to develop a tele-operated assistive robot for working at the hospital, except for some specific use cases. Tele-robotic is usually restricted to provide an effective remote supervision of the therapy at home after hospitalization or for surrogate clinicians passing medical consultations. This paper describes a novel cognitive architecture, which fulfills the requirements needed by a socially assistive robot used as therapist assistants in real hospital rehabilitation scenarios.

In order to correctly evaluate the validity and benefits of this proposal, future work will focus on testing our proposal in a long-term clinical use case conducted at the Hospital Universitario Virgen del Rocío (Seville): pediatric patients with upper limb motor deficit due to obstetric brachial plexus palsy and cerebral palsy. Both cases are among the most prevalent pathologies causing motor and cognitive deficits. Pediatric patients represent an interesting collective to be favored by these systems, as recent pioneering studies, including our own work, have shown that they can be easily driven into highly attentive and collaborating attitudes by letting them interact with social robots. Restricted to well-defined environments, the evolution of the project should be followed from the study of therapeutic sessions of increasing difficulty attending to the autonomy of the robot. From these pilot experiments, we will iteratively evaluate and improve the functionalities of THERAPIST, following a scheme that will require the active participation of engineers, therapists and patients within the loop, not only as designers or evaluators, but also as interactive partners. This evaluation will also require the definition of metrics and monitoring protocols to evaluate the progress of the patients, and the comparisons of the results with classical therapies.

\section{ACKNOWLEDGMENT}

This work has been partially granted by the Spanish Ministerio de Economía y Competitividad (MINECO) and FEDER funds under coordinated project no. TIN2012-38079-C03-01, TIN2012-38079-C03-02 and TIN2012-38079-C03-03

\section{REFERENCES}

[1] V. Alcázar, C. Guzmán, G. Milla, D. Prior, D. Borrajo, L. Castillo and E. Onaindía, PELEA: Planning, learning and execution architecture. In Proceedings of the 28th Workshop of the UK Planning and Scheduling Special Interest Group (PlanSIG'10), Brescia-Italy, 2010.

[2] J.P. Bandera, Vision-based gesture recognition in a Robot Learning by Imitation framework, $\mathrm{PhD}$ Thesis, Dpto. Tecnología Electrónica, Universidad de Málaga, 2010.

[3] R. Brooks, Intelligence without representation. Artificial Intelligence 47, 139-159, 1991.

[4] A. Clark, An embodied cognitive science?, Trends in Cognitive Sciences 3(9), 1999.

[5] J. Fasola and M. Mataric, Comparing physical and virtual embodiment in a socially assistive robot exercise coach for the elderly, Technical Report CRES-11-003, Center Robotics and Embedded Systems, University of Southern California, 2011.

[6] D. Feil-Seifer and M. Mataric, Ethical principles for socially assistive robotics, IEEE Robotics and Automation Magazine 18(1), 24-31, 2011.

[7] T. Fong, I. Nourbakhsh and K. Dautenhahn, A survey of socially interactive robots, Robotics and Autonomous Systems 42(3-4), 143-166, 2003.

[8] O. Holland, The Future of embodied artificial intelligence: Machine consciousness?. Embodied Artificial Intelligence, 37-53, 2004.

[9] S. Lemaignan, R. Ros, L. Mösenlechner, R. Alami and M. Beetz, ORO, A knowledge management module for cognitive architectures in robotics, Proc. of the IEEE/RSJ Int. Conf. on Intell. Robots and Systems, 2010.

[10] L. Manso, P. Bustos, P. Bachiller and M. Gutierrez, Graph grammars for active perception, Int. Conf. on Autonomous Robot Systems and Competitions, 63-68, 2012.

[11] M.J. Mataric, Socially assistive robotics, IEEE Intell. Syst. 21(4), 81-83, 2006.

[12] D. Reinkensmeyer, C. Pang, J. Nessler and C. Painter, Web-based telerehabilitation for the upper extremity after stroke, IEEE Trans. Neural Systems Rehabilitation Engineering 10(2), 102-108, 2002.

[13] C. Suárez Mejas, C. Echevarra, P. Nez, L. Manso, P. Bustos, S. Leal and C. Parra, Ursus: A Robotic Assistant for Training of Patients with Motor Impairments. Converging Clinical and Engineering Research on Neurorehabilitation, Edited by, J.L Pons, D. Torricelli and Marta Pajaro. Springer series on BioSystems and BioRobotics. Springer 2012. 